\title{
Proficiency Monitoring of Allergen Specific IgE macELISA-2018
}

\author{
Kenneth Lee ${ }^{* 1}$, Karen Blankenship ${ }^{1}$, Brennan McKinney ${ }^{1}$, Gerhard Kern ${ }^{2}$, Elizabeth Roth ${ }^{3}$, Janice Greenwood ${ }^{4}$, Santiago Cerrato ${ }^{5}$, \\ Laurent Drouet ${ }^{6}$ and Cecilia Tambone ${ }^{7}$
}

\author{
'Stallergenes Greer, 639 Nuway Circle, Lenoir, North Carolina, USA \\ ${ }^{2}$ IDEXX GmbH, Mörikestraße 28/3, D-71636 Ludwigsburg, Germany \\ ${ }^{3}$ IDEXX Laboratories, Inc. One IDEXX Drive, Westbrook, Maine, USA \\ ${ }^{4}$ IDEXX Laboratories, Inc. 1345 Denison Street, Markham, Ontario L3R 5V2, Canada \\ ${ }^{5}$ LETI s.l.u., Avda Cerdanyola, 92, 08172 Sant Cugat, Barcelona, Spain \\ ${ }^{6}$ Ceva Biovac, 6 rue Olivier de Serres, BP 10061 Angers Technopole, 49071 Beaucouzé, France \\ ${ }^{7}$ Agrolabo S.p.A.,Via Masero, 59, 10010 Scarmagno (TO), Italy
}

${ }^{*}$ Correspondence to: Kenneth W. Lee, PhD, Stallergenes Greer, 639 Nuway Circle, Lenoir, NC 28645, North Carolina, USA; Telephone: 828 759-7345, Fax: 828 754-7306; E-mail: ken.lee@stallergenesgreer.com

Received: September 18, 2018; Accepted: October 07, 2018; Published: October 14, 2018;

\begin{abstract}
The purpose of this study was to evaluate the reproducibility of results yielded using a macELISA for detection of allergen specific IgE in dogs and cats when run by nine different individuals in seven separate affiliated laboratories. Samples of 21 different sera were independently evaluated in each laboratory by differing operators in a single blinded fashion. For evaluations completed by multiple operators, the average inter-operator variance was calculated to be $5.2 \%$ (range $=2.0 \%-9.4 \%$ ). The average intra-assay variance among reactive assay calibrators in all laboratories was $4.4 \%$ (range $=1.5-$ 9.4\%). The overall inter-assay inter-laboratory variance evident with reactive calibrators was consistent among laboratories and averaged $6.9 \%$ (range $4.9-8.8 \%$ ). All laboratories yielded similar profiles and magnitudes of responses for replicate unknown samples; dose response profiles observed in each of the laboratories were indistinguishable. Correlation of EAU observed for individual allergens between and among all laboratories was strong ( $\mathrm{r}$ $>0.90, p<0.001)$. Collectively, the results demonstrated that ELISA for measuring allergen specific IgE is reproducible, and documents that consistency of results can be achieved not only in an individual laboratory, but among different operators and between laboratories using the same ELISA.
\end{abstract}

Keywords: IgE, ELISA, Proficiency, Atopy, Allergy, Immunotherapy,

\section{Introduction}

Stallergenes Greer maintains a proficiency monitoring program for laboratories that routinely run a monoclonal antibody cocktail based Enzyme-Linked Immunosorbent Assay ( $m a c$ ELISA) for evaluation of allergen specific IgE in serum samples [1-4]. The foundation for this program is based in the desire for inter-laboratory standardization and quality control measures that ensure the uniformity, consistency, and reproducibility of results among laboratories that perform the assays. This program is designed to periodically evaluate the proficiency of laboratories and ensures that individual operators yield consistent and reproducible results. The inaugural proficiency evaluations, initiated in 2009 and repeated in 2010, in six different laboratories documents that inter-laboratory standardization and quality control measures in the veterinary arena are on the immediate forefront and that uniformity, consistency, and reproducibility of results between laboratories is achievable [2]. Similarly, reproducibility of results among ten different laboratories was documented in the subsequent proficiency evaluations completed in 2013 and 2016 [3,4]. The results presented herein summarize the comparative results observed in the affiliate laboratories for the most recent proficiency evaluations that were completed in August 2018.

\section{Materials and Methods}

All serum samples, buffers, coated wells, calibrator solutions, and other assay components were aliquants of the respective lots of materials manufactured at Stallergenes Greer's production facilities (located in Lenoir, NC, USA) and supplied as complete kits to the participating laboratories along with the exact instructions for completing the evaluations.

\section{Participating Laboratories}

Seven independent Veterinary Reference Laboratories (VRLs) participated in the 2018 proficiency evaluation of macELISA. Participating laboratories included three separate IDEXX laboratories located in Memphis, Tennessee, Ludwigsburg, Germany, and Markham, Ontario Canada. Other affiliated European laboratories that participated in this evaluation included Agrolabo (Scarmagno, Italy), Laboratorios LETI (Barcelona, Spain), and Ceva Biovac (Beaucouzé, France). Stallergenes Greer Laboratories (Lenoir, NC) served as the 
prototype for evaluation of macELISA; the 2018 evaluations included results reported by three separate and independent operators. Because the performance characteristics of macELISA in Stallergenes Greer's VRL have been well documented for use over an extended period [1-4], all results observed in the other participating laboratories were compared directly with the results observed in Stallergenes Greer's reference laboratory.

\section{Serum Samples}

Separate pollen and mite reactive serum pools or non-reactive sera pools were prepared from cat and dog serum samples that previously had been evaluated using the macELISA for detection of allergen specific IgE in dogs and cats. The allergen specific reactivity of each sera pool ranged from nonreactive to multiple pollen or mite reactivity's. These sera pools and admixtures of the pools were used to construct a specific group of samples that exhibited varying reactivity to the allergens included in the evaluation panel. Eighteen samples were included in the blinded evaluation conducted by each laboratory. Two known pollen reactive control samples and one nonreactive control sample were also included; replicates of these identical samples were included as unknown blinded samples. Also included in the array of samples was a five tube three-fold serial dilution of a highly pollen-reactive pool, diluted into non-reactive sera, which served to document the dose response evident within the assay. The sera samples used for the current evaluation were identical to those evaluated during the 2016 proficiency testing [4]; all samples were stored at $-20^{\circ} \mathrm{C}$ for the interim between testing.

\section{Calibrators}

Grass pollen reactive calibrator solutions of predetermined reactivity in the macELISA were prepared as three-fold serial dilutions of a sera pool highly reactive to most pollen allergens. Replicates of each were evaluated in each assay run and served as a standard response curve for normalizing results observed with the various samples. All results were expressed as ELISA Absorbance Units (EAU) which is background-corrected observed responses expressed as milli absorbance.

\section{Buffers}

The buffers used throughout have been previously described [1-4], and included: a) well coating buffer: $0.05 \mathrm{M}$ sodium carbonate bicarbonate buffer, $\mathrm{pH}$ 9.6; b) wash buffer: Phosphate Buffered Saline (PBS), pH 7.4, containing $0.05 \%$ Tween 20 , and $0.05 \%$ sodium azide; c) serum and reagent diluent buffer: PBS, $\mathrm{pH} 7.4$, containing $1 \%$ fish gelatin, $0.05 \%$ Tween 20 and $0.05 \%$ sodium azide.

\section{Allergen Panel}

The allergen panel was a 24 allergen composite derived from the array of allergens that are included in the specific panels routinely evaluated in the various laboratories; the composite allergen panel consisted of 4 grasses, 6 weeds, 6 trees, 5 mites, and 3 fungi. The protocol for coating and storage of wells has been previously described [1-4].

\section{Sample Evaluations - macELISA}

The operational characteristics and procedures for the macELISAs have been previously described [1-4]. Following incubation of allergen coated wells with an appropriately diluted serum sample, allergen specific IgE is detected using a secondary antibody mixture of biotinylated monoclonal anti-IgE antibodies, streptavidin alkaline phosphatase as the enzyme conjugate, and $p$-nitrophenylphosphate $(p N P P)$ as substrate reagent. Specific IgE reactivity to the allergens is then estimated by determining the absorbance of each well measured at $405 \mathrm{nM}$ using an automated plate reader. All results are expressed as ELISA Absorbance Units (EAU) which are background-corrected observed responses expressed as milli absorbance [1].

\section{Statistics}

A coefficient of variation was calculated as the ratio of standard deviation and means of the responses observed for the calibrator solutions within different runs in multiple laboratories. Pearson's correlation statistic was used for inter-laboratory comparison among individual allergens.

\section{Results}

The assay variance (\% CV) observed with the calibrator solutions in the different laboratories are presented in Table 1 and are representative of the assay reproducibility in the various laboratories. The average intra-assay \% CV among positive calibrators (\#1-4) was $4.4 \%$ (range = 1.5-9.4\%); differences among laboratories or between assays and within assay runs were not detected. No substantial differences in results among various operators were revealed. The average interoperator variance documented for Stallergenes Greer technicians was calculated to be $5.2 \%$ (range $=2.0 \%-9.4 \%$ ). The average inter-assay variance (\% CV) observed in Stallergenes Greer's laboratory with the positive calibrators from multiple runs over a one year period has been documented at $8.9 \%$ (range $7.1 \%-9.7 \%$ ), and the inter-laboratory $\% \mathrm{CV}$ among reactive calibrators also remained relatively constant (average 12.1\%; range $11.2-13.4 \%$ )[1-3]. The results of the current evaluation (Table 1) are consistent with these published findings; the inter-assay variance among positive calibrators for all laboratories included in this evaluation was calculated to be $6.9 \%$ (range $=4.9$ $8.8 \%)$. Similar to these previously published studies, the intra-assay variability was higher with negative calibrator \#5 (average 3.9\%; range $2.4-8.9 \%$ ), and a similar increased intra-assay variability was evident with the background ODs (average 11.2\%; range 3.9-36.2\%).

To evaluate the strength of association with the magnitude of EAU results observed for each allergen among the different laboratories a Pearson's correlation coefficient was determined (Microsoft Excel 2016) for each laboratory pair. Because the macELISA is designed to yield comparable responses in dog and cat samples, comparison of results among affiliate laboratories included both cat and dog sample as a single population of sera samples. These results (Table 2) demonstrate that very high inter-laboratory correlation ( $r>$ $0.90 ; \mathrm{p}<0.001)$ is evident between the results observed in Stallergenes Greer's laboratory and those observed in six affiliate laboratories for all mites and pollen allergens. The correlation (Pearson's) of results 
observed with the fungal allergens within or between any of the testing laboratories was also substantial. However, the majority of results for the fungal allergens fell within the lower range of reactivity or within the negative range of the response curve ( $<150$ EAU). Consequently, the correlation of results among laboratories for the fungal allergens was somewhat less than the correlation evident with the mite and pollen allergens. The overall correlation of results observed in the various laboratories is summarized in Table 3; a very strong correlation was demonstrated between and among the results of the participating laboratories.

Table 1. Calculated variance of macELISA calibrator solutions observed with different laboratory runs by multiple operators during the 2018 Proficiency evaluation.

\begin{tabular}{|c|c|c|c|c|c|c|c|}
\hline \multirow{2}{*}{$\underline{\text { Variance }}$} & \multirow{2}{*}{$\mathrm{N}$} & \multicolumn{5}{|c|}{ Calibrator \% CV } & \multirow{2}{*}{$\begin{array}{c}\mathrm{BG}^{\dagger} \\
\% \mathrm{CV} \\
\end{array}$} \\
\hline & & $\# 1$ & \#2 & $\underline{\# 3}$ & $\# 4$ & $\underline{\# 5}$ & \\
\hline Inter-Laboratory & 238 & 6.2 & 10.3 & 9.7 & 9.6 & 6.9 & 17.2 \\
\hline Inter-Assay (Stallergenes Greer) & 84 & 4.9 & 8.8 & 8.0 & 6.1 & 2.8 & 21.9 \\
\hline \multicolumn{8}{|l|}{ Intra-Assay } \\
\hline Stallergenes Greer \#1 & 28 & 2.0 & 2.9 & 5.2 & 3.2 & 2.4 & 36.2 \\
\hline Stallergenes Greer \#2 & 28 & 4.5 & 9.4 & 8.5 & 4.6 & 2.8 & 5.3 \\
\hline Stallergenes Greer \#3 & 28 & 4.1 & 5.1 & 9.3 & 4.1 & 2.6 & 3.9 \\
\hline IDEXX Memphis & 28 & 8.1 & 3.0 & 3.6 & 2.7 & 2.5 & 5.2 \\
\hline IDEXX Canada & 28 & 2.1 & 4.9 & 5.1 & 4.3 & 4.3 & 8.2 \\
\hline IDEXX Germany & 28 & 1.5 & 4.1 & 2.8 & 3.4 & 3.8 & 7.7 \\
\hline Agrolabo & 28 & 3.5 & 7.1 & 6.2 & 6.5 & 8.9 & 16.3 \\
\hline Ceva Biovac & 28 & 3.3 & 5.0 & 3.8 & 2.7 & 3.8 & 6.4 \\
\hline LETI & 28 & 2.7 & 3.1 & 2.1 & 3.9 & 4.0 & 11.3 \\
\hline
\end{tabular}

"Calibrator \#1 is prepared as a dilution of a sera pool which is highly reactive to grass pollen allergens;

Calibrator $\# 5$ is a dilution of a negative sera pool.

Calibrators \#2 - \#4 are prepared as a serial 3-fold dilution of calibrator \#1.

${ }^{\dagger}$ Background responses observed with diluent in place of serum sample.

Because the sera samples included in the 2016 proficiency evaluation were identical to the samples included in the current proficiency evaluation (2018), the results observed for the 2016 proficiency evaluation in all laboratories were compared to the results observed within the 2018 proficiency evaluation. The results presented in Table 4 demonstrate strong intra-laboratory correlation among mite and pollen allergens. Consistent with the correlation of fungi responses within each of the proficiency evaluations, the correlation of the two proficiency evaluation comparison of fungi responses was somewhat reduced. Overall, a strong inter-laboratory correlation of results ( $>0.90)$ is also evident among the participating laboratories (Table 5) that completed the two proficiency evaluations.

There is no compelling evidence that the level of allergen specific IgE correlates with severity of clinical disease [5-8]. However, an evaluation that purports to measure allergen specific IgE should have a reduction in signal that is directly proportional to the dilution factor of the test ligand [9]. For an evaluation of the dose response in this ELISA, a five tube three-fold serial dilution of a highly pollen-reactive dog sera pool was included as unknown independent samples. To be expected, the magnitude of responses observed in each laboratory was reduced in direct proportion to dilution and the profile of response for each allergen was indistinguishable from the results presented for the previous proficiency evaluation completed in 2016 [4] (data not shown). Results from the final tube in the dilution scheme yielded results that were indistinguishable from negative responses, indicating a dilution extinction of detectable response.

\section{Discussion}

The results of the present study demonstrate that the variability between and among the affiliate laboratories and technicians are indistinguishable from the results evident within and between runs completed in the laboratory of Stallergenes Greer. The intra-assay variance observed with the positive calibrators evident among the various runs within each of the laboratories remains relatively low and indistinguishable among the various laboratories. Likewise, the interassay variance within each laboratory remained relatively constant and the results from all laboratories were demonstrably similar and the $\mathrm{CV}$ of the positive responses was relatively constant across the entire range of reactivity tested. Thus, we conclude that any and all laboratories and technicians are equally proficient in providing consistent results for all allergens tested and the results are well within the acceptable variance limits ( $\pm 20 \%$ ) established for this assay [1].

Comparison of results from the current proficiency evaluation (2018) with the results from the previous evaluation (2016), which evaluated identical samples, demonstrates multiple performance characteristic of the assay. The intra-assay comparison of the results for the two assays runs (Table 4) documents that comparable responses are yielded for mites and pollens in the two separate runs in each of the participating laboratories. The correlation of the two proficiency evaluation comparison of fungi responses was somewhat reduced. This less than stellar correlation is probably a result of the majority of the fungal responses falling within the negative range $(<150$ EAU). The overall correlation of results within the inter-laboratory comparison (Table 5) demonstrates the likelihood that the reported results for a specific sample between any two or among any and all laboratories will be indistinguishable. Further, similar results will be yielded with any specific sample that has been stored $\left(-20^{\circ} \mathrm{C}\right)$ for a period in excess of three years, indicating that the allergen specific IgE present in serum remains stable for at least this time period. The sera samples are the only component within the two assays runs that were from the identical manufacture lot; the coated wells and assay reagents used in the 2016 proficiency evaluation were from separate manufacture lots of the respective components used in the 2018 evaluations. Consequently, the results presented herein not only demonstrate stability of sera samples stored over an extended period ( $>3$ years), but that consistent results can be attained when differing lots of the interchangeable assay components are used. Indirectly, these results also substantiate that an adequate quality assurance/ quality control program has been established for the manufacture of the components and reagents included in this ELISA.

The lack of a regulatory mandated quality assurance program for serum allergen-specific IgE testing in companion animals, that independently monitors performance of all laboratories and assay formats, prompts Stallergenes Greer to accept the responsibility for continued evaluation of laboratories that routinely use the company's assays. Information presented herein documents the continued commitment of Stallergenes Greer and its affiliate laboratories to providing a stream of information relating these results to the veterinary community. 
Table 2. Inter-laboratory correlation of macELISA results observed with individual allergens in Stallergenes Greer Laboratory and the results observed in the individual affiliate laboratories.

\begin{tabular}{|c|c|c|c|c|c|c|}
\hline \multirow{2}{*}{ Allergens } & \multicolumn{6}{|c|}{$\begin{array}{c}\text { Inter-Laboratory Coefficient of Correlation* } \\
\text { Stallergenes Greer vs } \\
\end{array}$} \\
\hline & $\begin{array}{c}\text { IDEXX } \\
\text { Memphis }\end{array}$ & $\begin{array}{l}\text { IDEXX } \\
\text { Germany }\end{array}$ & $\begin{array}{l}\text { IDEXX } \\
\text { Canada }\end{array}$ & $\begin{array}{c}\text { Ceva } \\
\text { Biovac }\end{array}$ & $\underline{\text { Agrolabo }}$ & LETI \\
\hline \multicolumn{7}{|c|}{ 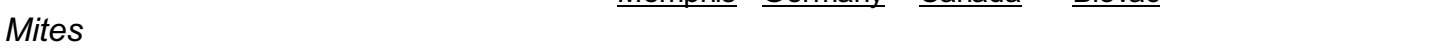 } \\
\hline Acaris siro & 0.998 & 0.999 & 0.998 & 0.985 & 0.987 & 0.991 \\
\hline Dermatophagoides farinae & 0.994 & 0.999 & 0.997 & 0.984 & 0.998 & 0.994 \\
\hline Dermatophagoides pteronyssinus & 0.994 & 0.998 & 0.995 & 0.994 & 0.978 & 0.980 \\
\hline Lepidoglyphus destructor & 0.985 & 0.994 & 0.976 & 0.965 & 0.931 & 0.952 \\
\hline Tyrophagus putrescentiae & 0.995 & 0.999 & 0.998 & 0.985 & 0.999 & 0.990 \\
\hline \multicolumn{7}{|l|}{ Grasses } \\
\hline June Grass (Poa pratensis ) & 0.997 & 0.997 & 0.997 & 0.984 & 0.986 & 0.983 \\
\hline Meadow fescue (Festuca pratensis ) & 0.998 & 0.998 & 0.997 & 0.987 & 0.990 & 0.983 \\
\hline Orchard Grass (Dactylis glomerata) & 0.999 & 0.998 & 0.997 & 0.988 & 0.992 & 0.986 \\
\hline Perennial Rye (Lolium perenne) & 0.997 & 0.998 & 0.997 & 0.988 & 0.989 & 0.986 \\
\hline \multicolumn{7}{|l|}{ Trees } \\
\hline Birch (Betula pendula) & 0.988 & 0.988 & 0.990 & 0.970 & 0.978 & 0.975 \\
\hline Cypress (Cupressus sempervirens) & 0.987 & 0.976 & 0.993 & 0.964 & 0.965 & 0.994 \\
\hline Hazelnut (Corylus avellana) & 0.988 & 0.989 & 0.991 & 0.968 & 0.984 & 0.962 \\
\hline Olive (Olea europaea) & 0.994 & 0.996 & 0.991 & 0.985 & 0.982 & 0.974 \\
\hline Populus mix (P. nigra, $P$. tremula, $P$. alba) & 0.994 & 0.994 & 0.991 & 0.984 & 0.977 & 0.975 \\
\hline Willow Black (Salix discolor) & 0.993 & 0.992 & 0.991 & 0.969 & 0.984 & 0.968 \\
\hline \multicolumn{7}{|l|}{ Weeds } \\
\hline English Plantain (Plantago lan & 0.995 & 0.996 & 0.995 & 0.987 & 93 & 0.982 \\
\hline Lambs Quarter (Chenopodium album) & 0.985 & 0.993 & 0.990 & 0.967 & 0.986 & 0.965 \\
\hline Mugwort (Artemisia vulgaris) & 0.996 & 0.993 & 0.992 & 0.982 & 0.990 & 0.970 \\
\hline Pellitory (Parietaria officinalis ) & 0.993 & 0.992 & 0.990 & 0.979 & 0.987 & 0.965 \\
\hline Ragweed (Ambrosia trifida, A. artemisiifolia) & 0.997 & 0.997 & 0.996 & 0.977 & 0.966 & 0.980 \\
\hline Sheep Sorrel (Rumex acetosella) & 0.996 & 0.993 & 0.994 & 0.986 & 0.986 & 0.978 \\
\hline \multicolumn{7}{|l|}{ Fungi } \\
\hline Alternaria alternata & 0.945 & 0.944 & 0.911 & 0.751 & 0.542 & 0.934 \\
\hline Aspergillus fumigatus & 0.968 & 0.958 & 0.951 & 0.968 & 0.893 & 0.984 \\
\hline Cladosporium herbarum & 0.963 & 0.959 & 0.976 & 0.954 & 0.762 & 0.971 \\
\hline Overall & 0.994 & 0.996 & 0.995 & 0.978 & 0.984 & 0.982 \\
\hline
\end{tabular}

"Pearson Correlation Coefficient ( $r)$; Good Correlation $(r>0.8, p<0.001)$

Table 3. Inter-laboratory correlation of macELISA results observed among individual affiliate laboratories.

\begin{tabular}{cccccccc}
\hline & \multicolumn{7}{c}{ Interlaboratory Coefficient of Correlation* } \\
\cline { 2 - 7 } Laboratory & $\begin{array}{c}\text { Stallergenes } \\
\text { Greer }\end{array}$ & $\begin{array}{c}\text { IDEXX } \\
\text { Memphis }\end{array}$ & $\begin{array}{c}\text { IDEXX } \\
\text { Germany }\end{array}$ & $\begin{array}{c}\text { IDEXX } \\
\text { Canada }\end{array}$ & $\begin{array}{c}\text { Ceva } \\
\text { Biovac }\end{array}$ & $\underline{\text { Agrolabo }}$ & LETI \\
Stallergenes Greer & 1 & 0.994 & 0.996 & 0.995 & 0.978 & 0.984 & 0.982 \\
IDEXX Memphis & 0.994 & 1 & 0.997 & 0.996 & 0.975 & 0.979 & 0.982 \\
IDEXX Germany & 0.996 & 0.997 & 1 & 0.997 & 0.982 & 0.984 & 0.987 \\
IDEXX Canada & 0.995 & 0.996 & 0.997 & 1 & 0.981 & 0.986 & 0.988 \\
Ceva Biovac & 0.978 & 0.975 & 0.982 & 0.981 & 1 & 0.985 & 0.993 \\
Agrolabo & 0.984 & 0.979 & 0.984 & 0.986 & 0.985 & 1 & 0.987 \\
LETI & 0.982 & 0.982 & 0.987 & 0.988 & 0.993 & 0.987 & 1 \\
\hline
\end{tabular}

"Pearson Correlation Coefficient (r); Good Correlation $(r>0.8, \mathrm{p}<0.001)$ 


\begin{tabular}{|c|c|c|c|c|c|c|c|}
\hline \multirow{2}{*}{$\underline{\text { Allergens }}$} & \multicolumn{7}{|c|}{$\begin{array}{c}\text { Inter-Laboratory Coefficient of Correlation } \\
2016 \text { vs } 2018 \text { Proficiency Samples } \\
\end{array}$} \\
\hline & $\begin{array}{l}\text { Stallergenes } \\
\text { Greer }\end{array}$ & $\begin{array}{c}\text { IDEXX } \\
\text { Memphis } \\
\end{array}$ & $\begin{array}{c}\text { IDEXX } \\
\text { Germany }\end{array}$ & $\begin{array}{r}\text { IDEXX } \\
\text { Canada } \\
\end{array}$ & Agrolabo & $\begin{array}{c}\text { Ceva } \\
\text { Biovac }\end{array}$ & $\underline{\text { LETI }}$ \\
\hline \multicolumn{8}{|l|}{ Mites } \\
\hline Acaris siro & 0.985 & 0.990 & 0.988 & 0.990 & 0.903 & 0.955 & 0.973 \\
\hline Dermatophagoides farinae & 0.985 & 0.972 & 0.984 & 0.985 & 0.981 & 0.933 & 0.972 \\
\hline Dermatophagoides pteronyssinus & 0.990 & 0.977 & 0.986 & 0.983 & 0.984 & 0.985 & 0.972 \\
\hline Lepidoglyphus destructor & 0.956 & 0.901 & 0.965 & 0.867 & 0.939 & 0.930 & 0.945 \\
\hline Tyrophagus putrescentiae & 0.960 & 0.977 & 0.971 & 0.978 & 0.960 & 0.933 & 0.934 \\
\hline \multicolumn{8}{|l|}{ Grasses } \\
\hline June Grass (Poa pratensis) & 0.995 & 0.991 & 0.996 & 0.986 & 0.981 & 0.985 & 0.988 \\
\hline Meadow fescue (Festuca pratensis) & 0.997 & 0.991 & 0.997 & 0.984 & 0.988 & 0.985 & 0.981 \\
\hline Orchard Grass (Dactylis glomerata) & 0.996 & 0.986 & 0.994 & 0.985 & 0.987 & 0.984 & 0.980 \\
\hline Perennial Rye (Lolium perenne) & 0.997 & 0.989 & 0.995 & 0.987 & 0.983 & 0.984 & 0.985 \\
\hline \multicolumn{8}{|l|}{ Trees } \\
\hline Birch (Betula pendula) & 0.973 & 0.930 & 0.965 & 0.957 & 0.972 & 0.973 & 0.975 \\
\hline Cypress (Cupressus sempervirens) & 0.978 & 0.917 & 0.969 & 0.977 & 0.982 & 0.969 & 0.947 \\
\hline Hazelnut (Corylus avellana) & 0.967 & 0.944 & 0.981 & 0.970 & 0.972 & 0.979 & 0.979 \\
\hline Olive (Olea europaea) & 0.978 & 0.954 & 0.972 & 0.958 & 0.941 & 0.959 & 0.963 \\
\hline Populus mix (P. nigra, $P$. tremula, $P$. alba) & 0.978 & 0.913 & 0.978 & 0.953 & 0.951 & 0.964 & 0.948 \\
\hline Willow Black (Salix discolor) & 0.959 & 0.941 & 0.962 & 0.943 & 0.949 & 0.944 & 0.949 \\
\hline \multicolumn{8}{|l|}{ Weeds } \\
\hline English Plantain (Plantago lanceolata) & 0.989 & 0.969 & 0.986 & 0.981 & 0.958 & 0.974 & 0.972 \\
\hline Lambs Quarter (Chenopodium album) & 0.991 & 0.978 & 0.986 & 0.963 & 0.968 & 0.966 & 0.974 \\
\hline Mugwort (Artemisia vulgaris) & 0.988 & 0.976 & 0.975 & 0.962 & 0.980 & 0.977 & 0.971 \\
\hline Pellitory (Parietaria officinalis ) & 0.993 & 0.967 & 0.990 & 0.982 & 0.974 & 0.991 & 0.977 \\
\hline Ragweed (Ambrosia trifida, A. artemisiifolia) & 0.987 & 0.983 & 0.981 & 0.971 & 0.969 & 0.962 & 0.956 \\
\hline Sheep Sorrel (Rumex acetosella) & 0.998 & 0.987 & 0.996 & 0.990 & 0.989 & 0.989 & 0.986 \\
\hline \multicolumn{8}{|l|}{ Fungi } \\
\hline Alternaria alternata & 0.930 & 0.942 & 0.878 & 0.883 & 0.640 & 0.780 & 0.945 \\
\hline Aspergillus fumigatus & 0.961 & 0.895 & 0.936 & 0.919 & 0.854 & 0.950 & 0.910 \\
\hline Cladosporium herbarum & 0.945 & 0.875 & 0.943 & 0.880 & 0.772 & 0.889 & 0.947 \\
\hline Overall & 0.972 & 0.960 & 0.971 & 0.963 & 0.947 & 0.940 & 0.956 \\
\hline
\end{tabular}

"Pearson Correlation Coefficient (r); Good Correlation ( $r>0.9, \mathrm{p}<0.001)$

Table 5. Inter-laboratory correlation of macELISA results observed among individual laboratories for evaluations completed in 2016 and 2018.

\begin{tabular}{cccccccc}
\hline \multirow{2}{*}{$\begin{array}{c}\text { Laboratory } \\
\text { 2016 Proficiency }\end{array}$} & \multicolumn{7}{c}{$\begin{array}{c}\text { Interlaboratory Coefficient of Correlation* } \\
\text { Laboratory - 2018 Proficiency }\end{array}$} \\
\cline { 2 - 8 } & $\begin{array}{c}\text { Stallergenes } \\
\text { Greer }\end{array}$ & $\begin{array}{c}\text { IDEXX } \\
\text { Memphis }\end{array}$ & $\begin{array}{c}\text { IDEXX } \\
\text { Germany }\end{array}$ & $\begin{array}{c}\text { IDEXX } \\
\text { Canada }\end{array}$ & Agrolabo & $\begin{array}{c}\text { Ceva } \\
\text { Biovac }\end{array}$ & $\underline{\text { LETI }}$ \\
Stallergenes Greer & 0.972 & 0.972 & 0.971 & 0.969 & 0.950 & 0.942 & 0.948 \\
IDEXX Memphis & 0.959 & 0.960 & 0.961 & 0.958 & 0.942 & 0.936 & 0.941 \\
IDEXX Germany & 0.971 & 0.971 & 0.971 & 0.970 & 0.952 & 0.945 & 0.950 \\
IDEXX Canada & 0.966 & 0.964 & 0.964 & 0.963 & 0.944 & 0.935 & 0.940 \\
Agrolabo & 0.966 & 0.965 & 0.966 & 0.965 & 0.947 & 0.938 & 0.945 \\
Ceva Biovac & 0.959 & 0.956 & 0.957 & 0.958 & 0.945 & 0.940 & 0.946 \\
LETI & 0.973 & 0.973 & 0.973 & 0.974 & 0.955 & 0.948 & 0.956 \\
\hline
\end{tabular}

"Pearson Correlation Coefficient (r); Good Correlation $(r>0.9, \mathrm{p}<0.001)$ 


\section{Authors Contributions}

Kenneth Lee contributed to the conception and design of the study; contributed to the acquisition, analysis, and interpretation of data; and drafted the manuscript. Karen Blankenship and Brennan McKinney manufactured all components used throughout the evaluation and contributed to acquisition of the data. Gerhard Kern, Elizabeth Roth, Janice Greenwood, Santiago Cerrato, Laurent Drouet, and Cecilia Tambone contributed to acquisition of the data. All authors gave final approval and agree to be accountable for all aspects of the work in ensuring that questions relating to the accuracy or integrity of any part of the work are appropriately investigated and resolved.

\section{Declaration of conflicting interests}

All authors from Stallergenes Greer are employees at Stallergenes Greer; other authors are employees of the affiliate laboratories.

\section{Funding}

Funding for this study was provided by Stallergenes Greer.

\section{References}

1. Lee KW, Blankenship KD, McCurry ZM, et al. (2009) Performance characteristics of a monoclonal antibody cocktail-based ELISA for detection of allergen-specific $\mathrm{IgE}$ in dogs and comparison with a high affinity IgE receptor-based ELISA. Vet Dermatol 20: 157-64. [crossref]

2. Lee KW, Blankenship KD, McCurry ZM, et al. 2012, Reproducibility of a Monoclonal Antibody Cocktail Based ELISA for Detection of Allergen Specific IgE in Dogs: Proficiency Monitoring of macELISA in Six US and European Laboratories. Vet. Immunol Immunopathol 148: 267-275.

3. Lee KW, Blankenship K, McKinney B, Kern G, Buch J, et al. (2015) Proficiency monitoring of monoclonal antibody cocktail-based enzyme-linked immunosorbent assay for detection of allergen-specific immunoglobulin E in dogs. Journal of Veterinary Diagnostic Investigation 27: 461-469.

4. Lee K, Blankenship K, McKinney B, Kern G, Roth E, et al. (2017) Continued Proficiency Monitoring Of Monoclonal Antibody Cocktail-Based Enzyme-Linked Immunosorbent Assay for Detection of Allergen Specific Immunoglobulin E in Dogs - 2016. Integr J Vet Biosci 1: 1-10.

5. DeBoer DJ, Hillier A (2001) The ACVD task force on canine atopic dermatitis (XVI): laboratory evaluation of dogs with atopic dermatitis with serum-based "allergy" tests. Vet Immunol Immunopathol 81: 277-87.

6. Gorman NT, Halliwell REW (1989) Atopic Diseases. In: Halliwell REW, Gorman NT (eds.). Veterinary Clinical Immunology. WB Saunders, Philadelphia, USA Pg No: 232-52.

7. Griffin CE, DeBoer DJ (2001) The ACVD task force on canine atopic dermatitis (XIV): clinical manifestation of canine atopic dermatitis. Vet Immunol Immunopathology 81: 255-69.

8. Griffin CE, Hillier A. (2001) The ACVD task force on canine atopic dermatitis (XXIV): allergen-specific immunotherapy. Vet Immunol Immunopathol 81: 363-83.

9. Tijssen P (1993) Processing of data and reporting of results of enzyme immunoassays. In: Burdon, RH, van Knippenberg PH (eds.). Practice and Theory of Enzyme Immunoassays. Elsevier, Amsterdam, Netherlands Pg No: 385-421.

\section{Citation:}

Kenneth Lee, Karen Blankenship, Brennan McKinney, Gerhard Kern, Elizabeth Roth, Janice Greenwood, Santiago Cerrato, Laurent Drouet and Cecilia Tambone (2018) Proficiency Monitoring of Allergen Specific IgE macELISA-2018. Integr J Vet Biosci Volume 2(3): 1-6. 